\title{
Differences between Estimation and Real Performance in School-Age Children: Fundamental Movement Skills
}

\author{
Gabriela Almeida, ${ }^{1,2}$ Carlos Luz, ${ }^{1,3}$ Rui Martins, ${ }^{1}$ and Rita Cordovil ${ }^{4}$ \\ ${ }^{1}$ Faculdade de Motricidade Humana, Universidade de Lisboa, Estrada da Costa, 1499-002 Cruz Quebrada-Dafundo, Portugal \\ ${ }^{2}$ Faculdade de Ciências da Saúde, Universidade Fernando Pessoa, Rua Delfim Maia 334, 4200-253 Porto, Portugal \\ ${ }^{3}$ Escola Superior de Educação de Lisboa, Instituto Politécnico de Lisboa, Campus de Benfica do IPL, 1549-003 Lisboa, Portugal \\ ${ }^{4}$ Laboratory of Motor Behavior, CIPER, Faculdade de Motricidade Humana, Universidade de Lisboa, Estrada da Costa, \\ 1499-002 Cruz Quebrada-Dafundo, Portugal \\ Correspondence should be addressed to Gabriela Almeida; galmeida@ufp.edu.pt
}

Received 9 April 2016; Accepted 20 June 2016

Academic Editor: Glenda Andrews

Copyright (C) 2016 Gabriela Almeida et al. This is an open access article distributed under the Creative Commons Attribution License, which permits unrestricted use, distribution, and reproduction in any medium, provided the original work is properly cited.

Observations in studies of estimation compared to actual performance in motor skills revealed that children are not always accurate and have a tendency to overestimate the maximum distance at which an action can be performed. The relationship between estimated and real motor competences was analyzed for several tasks: standing long jump (SLJ), throwing and kicking, and walking backwards (WB) on a balance beam. Children were asked to predict their maximum distance prior to performing those tasks. Participants were 303 children (160 boys), which were between 6 and 10 years of age $(M=8.63, S D=1.16)$. Children's estimations were compared with their real performance to determine their accuracy. Absolute error (|real performance - estimation|) and error tendency, that is, the direction of the error (overestimation, accuracy, and underestimation bias), were calculated. Children had a tendency to overestimate their performance and were more conservative in the WB task, a noncommon action. In general, it is possible to conclude that children, in the studied age span, tend to overestimate their performance, particularly in familiar skills. This fact may be determinant to the development of their motor competences, since they are more likely to engage and persist in motor tasks, but it might also be a problem in terms of child safety because it could increase the occurrence of unintended injuries.

\section{Introduction}

Children's perceived competence in the physical domain has attracted considerable interest in both motor skill development and sport psychology literatures. Perceived physical competence represents a psychological judgment about children's perception of how able they are in the physical domain [1]. Higher perceived competence is related with motor skill proficiency and increased levels of physical activity [2]. The overestimation of children's capabilities may have a positive effect on engaging them in motor activities and sports $[1,3]$ that improve motor proficiency. It should be noted, however, that, within Harter's theory, the measure of perceived physical competence is not obtained directly by doing the physical task.
The relationship between children's perception and real motor skill competence, obtained by a direct measure of performance, in order to ascertain whether the perceptual estimation reflects accuracy of the limits of their action capabilities, is a different line of research, based on Gibson's ecological approach $[4,5]$ to perception and action. This approach has provided the theoretical framework to the studies on the perception of judgments in actions capabilities. A central concept of Gibson's theory of direct perception is affordance, defined as the intrinsic relationship between a person's action capabilities and the properties of the environment, that is, the opportunities and dangers which the person perceives while acting in the environment [4]. During development children learn to perceive their opportunities for action, or affordances, in different environments. 
The environment is challenging for children and taking risks, testing, and experiencing different motor skills are part of children's development. The environment provided positive and negative affordances, that is, environment opportunities or environment dangers [4].

Although perceived motor competence is an important psychological construct, it might not have a direct relation with children's ability to estimate their motor competence in task-specific activities. Gabbard et al. [6] conducted a study with 7-9- and 11-year-old children to examine the relationship between the estimation of reachability and the perceived motor competence. The authors [6] suggested that the perceived motor competence, as a general measure based on a psychological construct, may "not reflect the intentions or real motor abilities" (pg. 156) and suggest more studies "tied to context-specific measures of perceived abilities in relation to the specificity of the task" (pg. 157). However, literature studies assessing children's real and perceived motor competence using the same skills, as tasks of real skill ability, are scarce. As for research based on Harter's theory, the studies that analyzed the estimation of action capabilities with a match task tend to empirically support that, in general, children are less accurate than adults, exhibiting the tendency to overestimate the limits of their ability [7, 8]. Adults are more accurate than children, and younger children are less accurate than older children. The accuracy of children's perception improves with age and cognitive development $[1,3]$. According to Harter's theory, it is considered normal for children to overestimate their motor competence because of cognitive limitations that make it difficult to distingue between their ideal and their real ability [1]. Plumert's study [7] also considered how experience influenced the accuracy of estimations. When children were given experience with the tasks before the test trials, 8 -year-old children but not 6-yearolds benefited from experience.

Children use their motor repertoires to engage in various physical activities, sport, and games across their lifespan. This repertoire is developed as a result of the combination of many factors such as experience, motor competences, and environmental or individual constraints. In this motor repertoire, fundamental movement skills (FMS) are the main skills that children with ages 2-7 years are expected to improve, to achieve a proficient level. These are gradually combined in a variety of ways to become sport skills [9]. Fundamental movement skills should persist for most part of the lifespan and are commonly categorized as fundamental locomotor skills (e.g., running, jumping, and hooping), fundamental manipulative skills (e.g., throwing, catching, and kicking), and fundamental stability skills (e.g., dynamic and static balance). Locomotor and manipulative movement skills engage an element of dynamic balance [10]. The mastery of fundamental movement skills is essential for the acquisition of more advanced, specific, and refined movement activities. In addition, a greater perceived motor competence in FMS has been related with the future adoption of active and healthier lifestyles [2].

Within the scope of an ecological perspective to perception and action, and based on the findings in the field of childhood, the specific aim of this study was to provide further assessment of the systematic overestimation of estimated competence reported in the literature $[7,11,12]$. In addition, we aimed at gathering information on the following: (i) How accurate are children in estimating their movement skills? (ii) Is there a gender difference in the accuracy of estimations? (iii) Does the accuracy in estimations differ for different fundamental movement skills (locomotor, manipulative, and dynamic balance tasks)? Following the suggestion of evaluating perceived motor competence using contextspecific measures, instead of a general measure based on a psychological construct [6], the purpose of this research was to directly examine the estimation of fundamental movement skills in children and to compare it with their real performance.

\section{Methods}

2.1. Participants. A sample of 303 children ( 160 boys and 143 girls), with ages between 6.48 years and 10.93 years $(\mathrm{M}=$ 8.63 years; $\mathrm{SD}=1.16$ ), participated in the study. Ethics approval was granted from the Ethics Council of Faculty of Human Kinetics, University of Lisbon. Prior informed consent from the parents and verbal assent from the children were obtained.

2.2. Measures and Procedures. We have performed assessments based on the same experimental paradigm: firstly children were asked to predict their ability, and secondly they were asked to perform those same tasks. No feedback from the evaluator or from the outcome of the actual performance was given to the children. The SLJ performance was measured following standard procedures $[13,14]$. The child was instructed to jump as far as possible from a standing start with feet slightly apart. The test was performed twice and the best of the 2 attempts (measured in $\mathrm{cm}$ ) was used for analysis. Before performing the SLJ, the child was asked to estimate his/her maximum jumping distance. During this estimation, the participant stood behind a line, while the evaluator starting at the feet of the child, slowly, and steadily unraveled a measuring tape until the child told her to stop, indicating the maximum estimated distance of jump (see Figure 1). The child was allowed to make fine adjustments after the order to stop if he/she found it necessary. The task was conducted in a uniform floor with no marks that could help the child to memorize the estimated location.

For the throwing condition, a mini soccer goal $(120 \mathrm{~cm} \times$ $80 \mathrm{~cm}$ ) was placed $1 \mathrm{~m}$ above the floor on a table, and a softball was used. For the kicking condition, the mini soccer goal was placed on the floor, and a size 4 soccer ball was used. The floor was marked every $2 \mathrm{~m}$, from $2 \mathrm{~m}$ to $20 \mathrm{~m}$ away from goal. In both tasks, the child stood upright in front of the goal and behind the $20 \mathrm{~m}$ line. From this position, the child was asked to go to the mark that he/she estimated to be the maximum distance to successfully throw/kick the ball into to the mini soccer goal. This distance was registered as the child's estimation. After that, the evaluator asked the child to throw/kick the ball into the target. If the child succeeded, he/she was asked to throw/kick from a farther line. This procedure was repeated until the child failed the target. When 


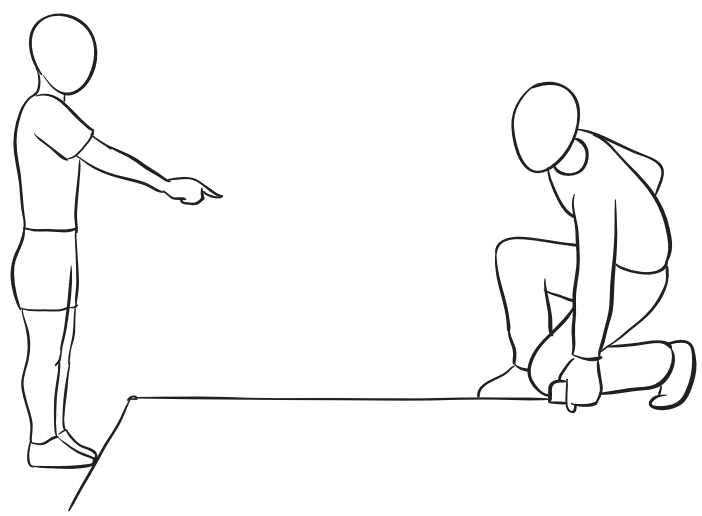

(a)

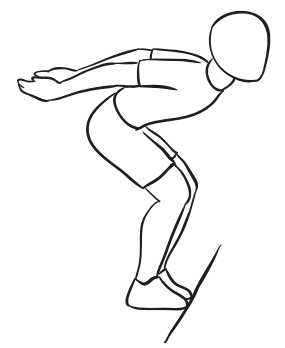

FIgURE 1: Estimation (a) and performance (b) of the standing long jump task.

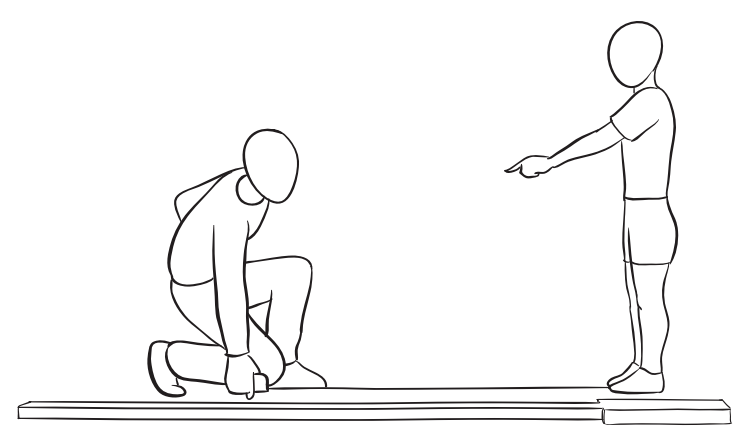

(a)

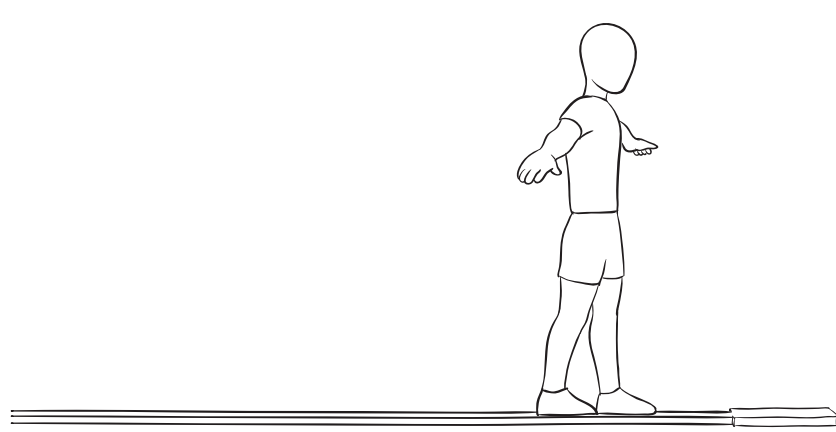

(b)

FIGURE 2: Estimation (a) and performance (b) of the walking backwards balance task.

the child failed (in any throw/kick position), he/she was asked to throw/kick from a closer line. This procedure was repeated until the child succeeded. The final successful position was the real distance recorded.

Participants also performed a balance task in which they walked backwards along a balance beam, $6 \mathrm{~cm}$ wide, $3 \mathrm{~cm}$ high, and $3 \mathrm{~m}$ long, without stepping off the beam. Children estimated how far they could walk backwards before performing the task. Once the participants indicated they understood the procedure, the estimation judgment was collected. The observer asked the children to estimate the farthest distance they could walk backwards before performing the task. The observer slowly unraveled a measuring tape until the child told her to stop. This measurement corresponded to child's estimated maximum WB. The child was allowed to fine-tune the measurement until she/he was satisfied. The estimation task was performed from the starting position in the standing front upright posture, after which the child turned and performed the real action backwards (see Figure 2). The task was performed twice and the best score (measured in $\mathrm{cm}$ ) was used for analysis.

2.3. Data Collection and Analysis. Absolute error and error tendency [15] of the SLJ, kicking, throwing, and WB tasks were analyzed. Absolute error is defined as the difference between the real performance and the estimation (|real performance - estimation|). Absolute error indicates the discrepancy in centimeters (SLJ and WB) or in meters (throwing and kicking) between estimation and real motor performance. Absolute error measures the error magnitude but not the under- or overestimation bias. Error tendency (i.e., frequency of overestimation, accuracy, and underestimation bias) indicates the direction of the error. For the jumping and WB tasks, a $\pm 12 \mathrm{~cm}$ error was allowed for estimations to be considered accurate. This value was settled by taking the average variability of the SLJ data (SD = 25.42) and the children's foot size as criteria. Considering this, an overestimation occurred when the estimation was more than $12 \mathrm{~cm}$ above that of the real performance and an underestimation occurred when the estimation was less than $12 \mathrm{~cm}$. Independent samples $t$-tests were used to compare differences in the real performance and in the error variable (absolute error) between genders. Pearson's chisquare tests were used to determine differences in error tendency according to gender. Pearson correlations were used to examine relationships between the variables (real performance, estimation, and chronological age). The level of significance for statistical analyses was set at $p<0.05$. Data analyses were conducted using SPSS (version 21). 


\section{Results}

3.1. Standing Long Jump. The maximum distance in SLJ was significantly greater in boys $(\mathrm{M}=128.36, \mathrm{SD}=24.49)$ than in girls $(\mathrm{M}=115.85, \mathrm{SD}=24.89)(t(301)=4.40, p<0.001)$. There was a significant and positive relationship between age and maximum SLJ ( $r=0.33, p<0.001)$. After separating by gender, there was a significant association between boys' age and real SLJ ( $r=0.40, p<0.001)$, and girls' chronological age and real SLJ $(r=0.30, p<0.001)$.

Correlation analysis showed that there was a significant and positive association between SLJ and the estimation of SLJ $(r=0.37, p<0.001)$. After separating by gender, there was a significant but weak association between boys' estimated and real SLJ $(r=0.37, p<0.001)$ and girls' estimated and real SLJ $(r=0.25, p=0.003)$.

No statistically significant gender differences were observed in absolute error $(t(301)=0.42, p=0.68)$ (boys: $\mathrm{M}=29.36, \mathrm{SD}=25.80$; girls: $\mathrm{M}=28.13, \mathrm{SD}=25.50)$.

Most children $(56.11 \%)$ have a tendency to overestimate their jump performance (see Table 1). About 28.05\% of the children are able to accurately estimate their jump and $15.84 \%$ underestimate it. A scatter plot of children's estimation and real SLJ is presented in Figure 3. The results show a significant association between the error tendency and gender $\left(\chi^{2}(2)=\right.$ 10.45, $p=0.005)$. Despite both genders showing an overestimation tendency, this tendency is slightly greater in boys (63.13\% versus $48.25 \%$ ), and less boys than girls underestimated their action capabilities (22.38\% versus $10.00 \%)$.

3.2. Throwing and Kicking. On average, boys showed a greater maximum throwing distance $(\mathrm{M}=5.53, \mathrm{SD}=2.89)$ than girls $(\mathrm{M}=3.99, \mathrm{SD}=2.16)(t(291,78)=5.28, p<0.001)$. The maximum kicking distance was also greater in boys $(\mathrm{M}=$ $7.06, \mathrm{SD}=3.87)$ than in girls $(\mathrm{M}=5.50, \mathrm{SD}=2.97)$ $(t(294,37)=3.97, p<0.001)$. Children's estimations and real performances were found to be significantly and positively associated (throwing: $r=0.52, p<0.001$; kicking: $r=0.60$, $p<0.001$ ). After separating by gender, there was a significant association between boys' estimated and real competence (throwing: $r=0.53, p<0.001$; kicking: $r=0.59, p<0.001$ ) and girls' estimated and real competence (throwing: $r=0.34$, $p<0.001$; kicking: $r=0.55, p<0.001)$. There was also a significant but weak relationship between the age and with both tasks: throwing $(r=0.25, p<0.001)$ and kicking $(r=0.17, p=0.003)$.

After separating by gender, there was a significant association between boys' age and real throwing $(r=0.34, p<$ $0.001)$ and girls' age and real throwing $(r=0.18, p=0.03)$. The kicking skill was also related with boys' age $(r=0.24$, $p=0.03)$. There was no significant association between girls' age and real kicking $(r=0.11, p=0.18)$.

Regarding absolute error, girls were more accurate than boys in both throwing $(t(289,201)=2.04, p=0.04)$ and kicking tasks $(t(291,76)=3.53, p<0.001)$ (throwing: boys: $\mathrm{M}=3.40, \mathrm{SD}=3.03$; girls: $\mathrm{M}=2.78, \mathrm{SD}=2.29$; kicking: boys: $\mathrm{M}=3.39, \mathrm{SD}=2.80$; girls: $\mathrm{M}=2.39, \mathrm{SD}=2.08$ ).

Children had a clear tendency to overestimate the maximum distance achieved in both manipulative tasks: $73.60 \%$ of

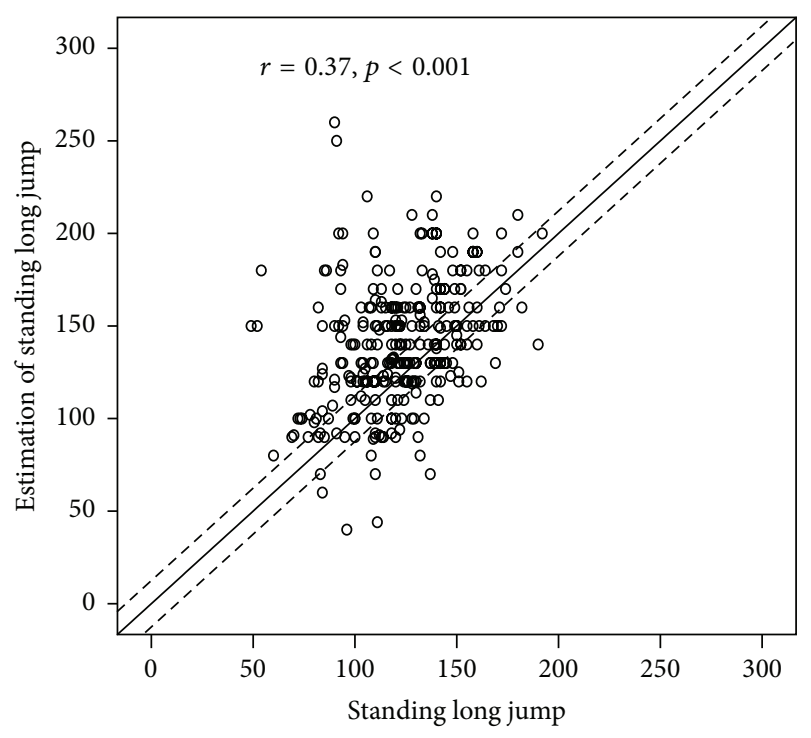

FIGURE 3: Scatter plot of children's estimation and real standing long jump (in $\mathrm{cm}$ ). The continuous line represents perfect agreement between estimation and real standing long jump $(n=13)$. The dashed lines indicate the interval within which the jump is considered accurate $( \pm 12 \mathrm{~cm})$. Estimations above the superior dashed line were considered overestimations. Estimations below the bottom dashed line were considered underestimations.

the children overestimated their throwing ability and $63.37 \%$ overestimated their kicking ability (see Table 1). There was no significant association between the error tendency and gender $\left(\chi^{2}(2)=0.07, p=0.97\right.$ (throwing); $\chi^{2}(2)=0.20$, $p=0.91$ (kicking)).

3.3. Walking Backwards. Concerning the walking backwards task, although girls ( $\mathrm{M}=201.91, \mathrm{SD}=107.99)$ performed, on average, better than boys $(M=195.64, S D=102.42)$, this was not statistically significant $(t(301)=-0.52, p=$ $0.60)$. There was a significant but weakly association between chronological age and maximum WB $(r=0.29, p<0.001)$. After separating by gender, there was a significant association between boys' age and real WB $(r=0.31, p<0.001)$ and girls' age and real WB $(r=0.28, p=0.001)$.

Children's estimations and real performances were positively but weakly correlated $(r=0.20, p<0.001)$. When dividing by gender, there was a significant but weak association between boys' estimated and real WB $(r=0.25$, $p=0.002)$ and girls' estimated and real WB $(r=0.17$, $p=0.04)$.

Concerning the absolute error, boys $(\mathrm{M}=87.24, \mathrm{SD}=$ 77.55) and girls $(\mathrm{M}=93.34, \mathrm{SD}=79.67)$ were not significantly different $(t(301)=-0.66, p=0.50)$.

Children have a tendency to overestimate their WB performance (45.21\%) (see Table 1). About 29.05\% of the children tend to underestimate and $25.74 \%$ are able to accurately estimate their WB. Boys have more tendency to overestimate ( $47.50 \%$ versus $42.66 \%$ ) their WB skills, whereas girls tend to underestimate (32.17\% versus $26.25 \%$ ); however this difference was not significant $\left(\chi^{2}(2)=1.034, p=0.52\right)$. 


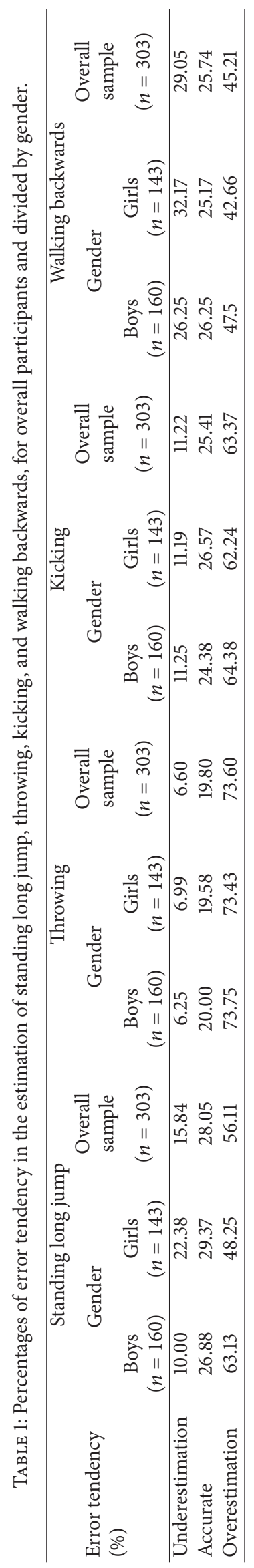




\section{Discussion}

The findings indicate that boys perform significantly better than girls, except for the WB, children's real and estimated performances are positively associated, but the association is only weak to moderate, children exhibit an overestimation bias for the four assessed skills, boys show an overestimation tendency, while for the SLJ, underestimation is more frequent in girls, and children are more conservative in the WB task, a noncommon action.

The gender differences in real performance could be explained by the interaction of biological and environmental influences, since gender differences in the biological characteristics, such as height, weight, and muscle mass, are minimal during childhood [16]. On the other hand, boys are more physically active than girls $[17,18]$ and are more engaged in opportunities and experiences that refine their motor skills competence.

Liong et al. [19] found that children's perceived and actual locomotor skills, assessed by six locomotor skills (running, horizontal jumping, hopping, sliding, leaping, and galloping), were not significantly associated $(r=0.03)$. In our study, we found that children's, boys' and girls' estimated and real locomotor competence (assessed through the SLJ) were weakly significantly associated. We have also found that real throwing and kicking competences are positively associated with children's estimated competences. This is in agreement with Barnett et al. [20] and Liong and coauthors [19] where, similarly to our study, the perceived competence items were shown to be direct reflection of the motor competence. Additionally, we have found a significant correlation between estimated and real skills for both genders. Liong and colleagues [19] found that boys' perception was associated with their actual object control competence $(r=0.26)$ but, contrary to our results, the association was not significant for girls. Barnett and coauthors [20] found that actual object control competence (six perceived skills) was associated with perceived competence, and this relationship did not differ with gender.

Previous research has suggested that children overestimate their action capabilities for different FMS [7, 11, 21]. Plumert [7] suggested two factors that may play a role in the overestimation of abilities. The first concerns the attractiveness of the goal. The second may be related to the way the tasks are conducted, where the fact that there is no bodily penalty may lead to an overestimation of the abilities. Children might have overestimated their performance, more than they would in real life, due the safe environment provided and the low possibility of harm. Whereas $45.21 \%$ of the children overestimate the WB and $56.11 \%$ the jumping, $63.37 \%$ and $73.60 \%$ of the children overestimate their kicking and throwing abilities, respectively. One possible reason for this discrepancy is the familiarity of the skill. For the less common skill, the walking backwards task, the percentage of overestimation was lower, when compared with the manipulative skills. There was also a higher percentage of underestimation $(29.05 \%)$ in WB. The unfamiliarity of the task may have contributed to a more conservative judgment, when compared to the other actions. In addition, throwing and kicking a ball into a soccer goal do not involve risk. The motivation of participant, the influence of specific motor experience, the type of task, the presence of any allocentric frame of reference, and the number of trials allowed per task are some of the factors that may have contributed to the discrepancy between estimated and actual FMS. The influence of these variables should be further explored in future studies.

The gender differences in estimation of physical abilities have not yet been documented. Instead, the focus of the available studies is on perceived motor competence as a psychological construct, with boys demonstrating higher perceived motor competence scores than girls [3]. In addition, it has been found that boys' perception was associated with their real object control ability [19]. We found evidences that girls are more likely to underestimate their SLJ capability than boys, whereas the boys tend to overestimate their capability more frequently than girls. For the other tasks, no differences in the error tendency were found between genders. Boys are known to be slightly more active, to obtain more pleasure from high-intensity stimuli, and to engage in active roughand-tumble, whereas girls display a stronger ability to manage and regulate their attention and inhibit their impulses [22]. According to previous studies [12,23], impulsive and highly active children are more likely to overestimate their abilities. Although quite speculative, one possible explanation for the results of this study is perhaps that the temperament of children is influenced by gender. That is, gender differences in temperament such as attention, impulsivity, and inhibitory control, amongst other factors, may play a role in the accuracy of the child's judgments about its own physical abilities. The fact that girls showed a more cautious estimation of their real ability might reduce the occurrence of negative consequences of an overestimation but, on the other hand, it is also possible that they do not refine knowledge of the limits of their own action capabilities.

\section{Conclusion}

The goal of this study was to investigate the relationship between estimated motor performance and real motor performance, in children from ages 6 to 10 years, on a set of fundamental movement skills tasks (SLJ, throwing, kicking, and WB). The novelty of this study is that the assessment of estimated performance exactly matches the assessment of real fundamental movement skills performance. Children tended to systematically overestimate their FMS, the more so for the fundamental manipulative skills than for the fundamental stability skill. In addition, children's real and estimated performances were significantly associated with the four studied skills. The overestimation of children's capabilities may have a positive effect on engaging in movement activities, sports, and play that improve motor proficiency but can also result in negative effects if children place themselves at risk of unintended injury. The results of this investigation highlight the importance of giving children opportunities to practice and estimate their motor proficiency. During childhood it is important to promote movement activities for children with the aim of enhancing the development of 
fundamental movement skills but also the ability to make more accurate judgments about their own physical abilities. Given the established association between the overestimation tendency and accidental injuries in children [7], intervention programs, which provide developmentally appropriate experiences and opportunities, targeting to improve judgments accuracy, should be considered at all ages. The results of this study should raise awareness of professionals working with children. The results highlight the importance of giving children opportunities to practice and estimate their motor proficiency. A more accurate perception of children's abilities will probably prevent and reduce unintentional injuries, which might occur during their participation in sports or other activities, such as playing at home or in playgrounds.

\section{Competing Interests}

The authors declare that they have no competing interests.

\section{Acknowledgments}

The authors would like to thank all of the children and their parents who have participated in their research. Thanks are due to Filipe Alves for drawing the illustrations.

\section{References}

[1] Harter, The Construction of the Self: A Developmental Perspective, The Gilford Press, New York, NY, USA, 1999.

[2] D. F. Stodden, J. D. Goodway, S. J. Langendorfer et al., "A developmental perspective on the role of motor skill competence in physical activity: an emergent relationship," Quest, vol. 60, no. 2, pp. 290-306, 2008.

[3] S. Harter, "The perceived competence scale for children," Child Development, vol. 53, no. 1, p. 87, 1982.

[4] J. J. Gibson, "The theory of affordances," in Perceiving, Acting and Knowing: Toward an Ecological Psychology, R. Shaw and J. Bransfard, Eds., pp. 69-81, Lawrence Erlbaum Associates, 1977.

[5] J. J. Gibson, The Ecological Approach to Visual Perception, 1979.

[6] C. Gabbard, P. Cačola, and A. Cordova, "Is perceived motor competence a constraint in children's action planning?" Journal of Genetic Psychology, vol. 170, no. 2, pp. 151-158, 2009.

[7] J. M. Plumert, "Relations between children's overestimation of their physical abilities and accident proneness." Developmental Psychology, vol. 31, no. 5, pp. 866-876, 1995.

[8] P. Rochat, "Perceived reachability for self and for others by 3- to 5-year-old children and adults," Journal of Experimental Child Psychology, vol. 59, no. 2, pp. 317-333, 1995.

[9] D. Gallahue and J. Ozmun, Understanding Motor Development: Infants, Children, Adolescents, Adults, McGraw-Hill, Boston, Mass, USA, 6th edition, 2006.

[10] D. L. Gallahue and F. Cleland-Donnelly, Developmental Physical Education for all Children, Human Kinetics, Champaign, Ill, USA, 4th edition, 2007.

[11] D. C. Schwebel and M. L. Bounds, "The role of parents and temperament on children's estimation of physical ability: links to unintentional injury prevention," Journal of Pediatric Psychology, vol. 28, no. 7, pp. 505-516, 2003.
[12] J. M. Plumert and D. C. Schwebel, "Social and temperamental influences on Children's overestimation of their physical abilities: links to accidental injuries," Journal of Experimental Child Psychology, vol. 67, no. 3, pp. 317-337, 1997.

[13] L. M. Chung, L. P. Chow, and J. W. Chung, "Normative reference of standing long jump indicates gender difference in lower muscular strength of pubertal growth," Health, vol. 5, no. 6, pp. 6-11, 2013.

[14] S. Gontarev, V. Zivkovic, L. A. Velickovska, and M. Naumovski, "First normative reference of standing long jump indicates gender difference in lower muscular strength of Macedonian school children," Health, vol. 6, no. 1, pp. 99-106, 2014.

[15] R. Cordovil and J. Barreiros, "Egocentric or allocentric frameworks for the evaluation of other people's reachability," Human Movement Science, vol. 30, no. 5, pp. 976-983, 2011.

[16] K. Haywood and N. Getchell, Life Span Motor Development, Human Kinetics, Champaign, Ill, USA, 2009.

[17] K. Finn, N. Johannsen, and B. Specker, "Factors associated with physical activity in preschool children," Journal of Pediatrics, vol. 140, no. 1, pp. 81-85, 2002.

[18] R. R. Pate, K. A. Pfeiffer, S. G. Trost, P. Ziegler, and M. Dowda, "Physical activity among children attending preschools," Pediatrics, vol. 114, no. 5, pp. 1258-1263, 2004.

[19] G. H. E. Liong, N. D. Ridgers, and L. M. Barnett, "Associations between skill perceptions and young children's actual fundamental movement skills," Perceptual and Motor Skills, vol. 120, no. 2, pp. 591-603, 2015.

[20] L. M. Barnett, N. D. Ridgers, and J. Salmon, "Associations between young children's perceived and actual ball skill competence and physical activity," Journal of Science and Medicine in Sport, vol. 18, no. 2, pp. 167-171, 2015.

[21] C. Gabbard, P. Caçola, and A. Cordova, "Does general motor imagery ability (via questionnaire) predict estimation of reachability in children?" Journal of Imagery Research in Sport and Physical Activity, vol. 3, no. 1, pp. 1-12, 2008.

[22] N. M. Else-Quest, J. S. Hyde, H. H. Goldsmith, and C. A. Van Hulle, "Gender differences in temperament: a meta-analysis," Psychological Bulletin, vol. 132, no. 1, pp. 33-72, 2006.

[23] D. C. Schwebel and J. M. Plumert, "Longitudinal and concurrent relations among temperament, ability estimation, and injury proneness," Child Development, vol. 70, no. 3, pp. 700-712, 1999. 


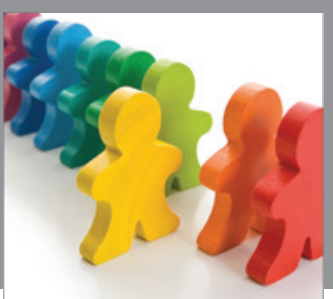

Autism

Research and Treatment
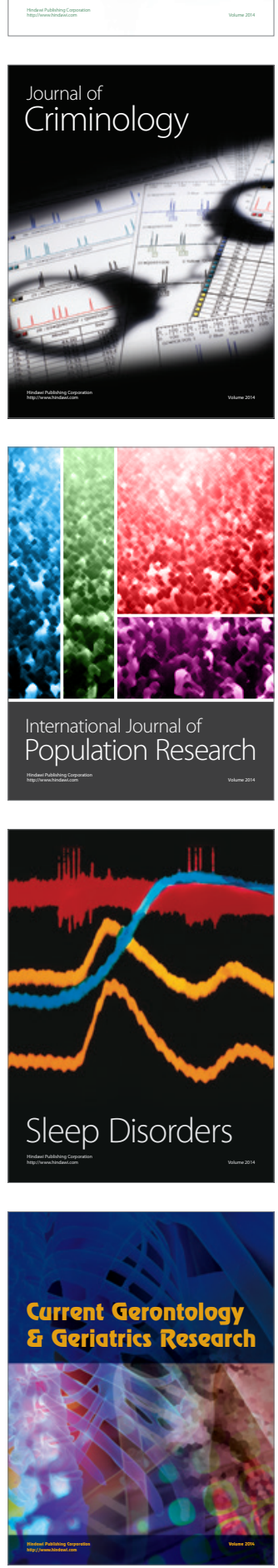

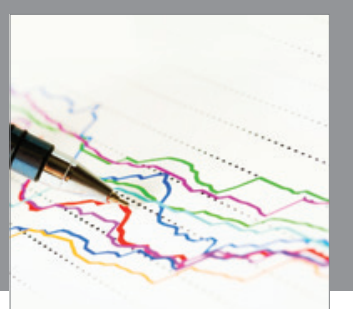

Economics

Research International
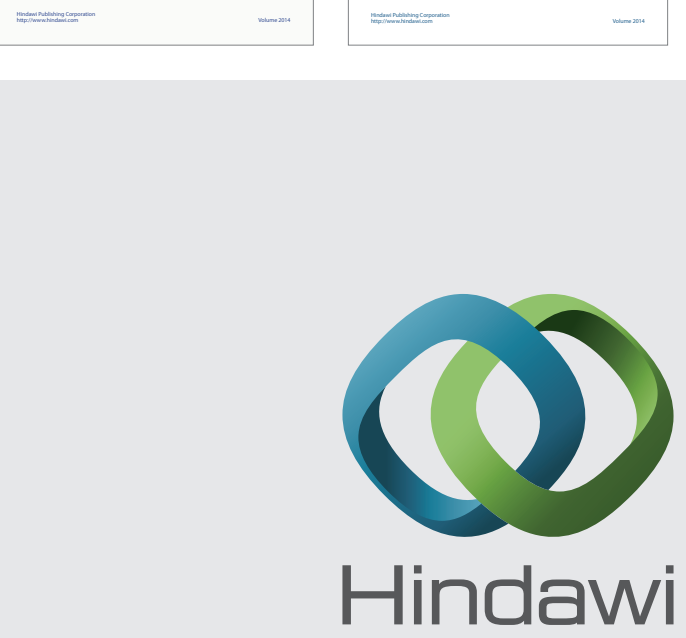

Submit your manuscripts at

http://www.hindawi.com
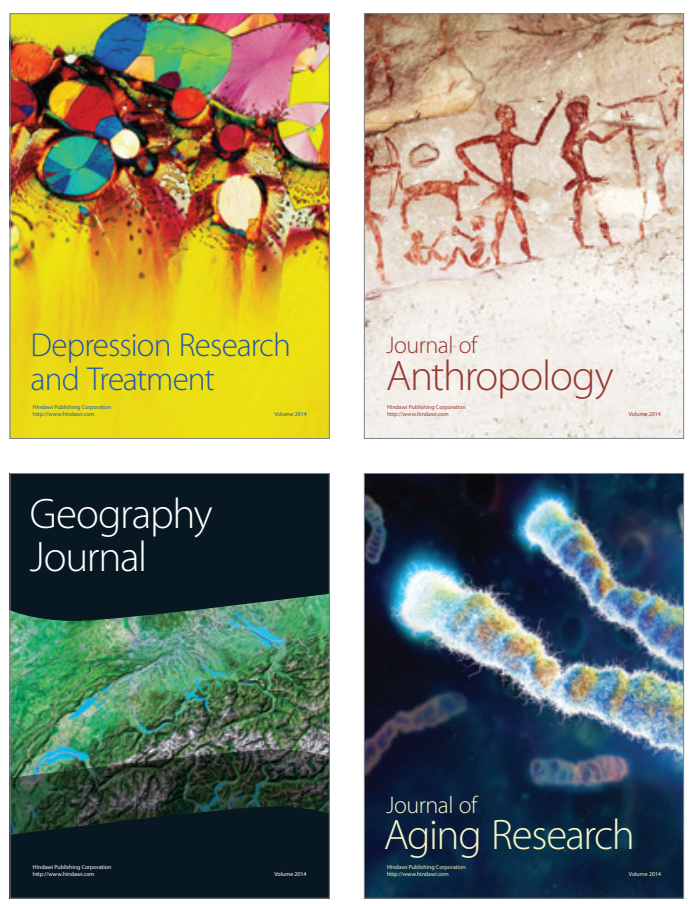
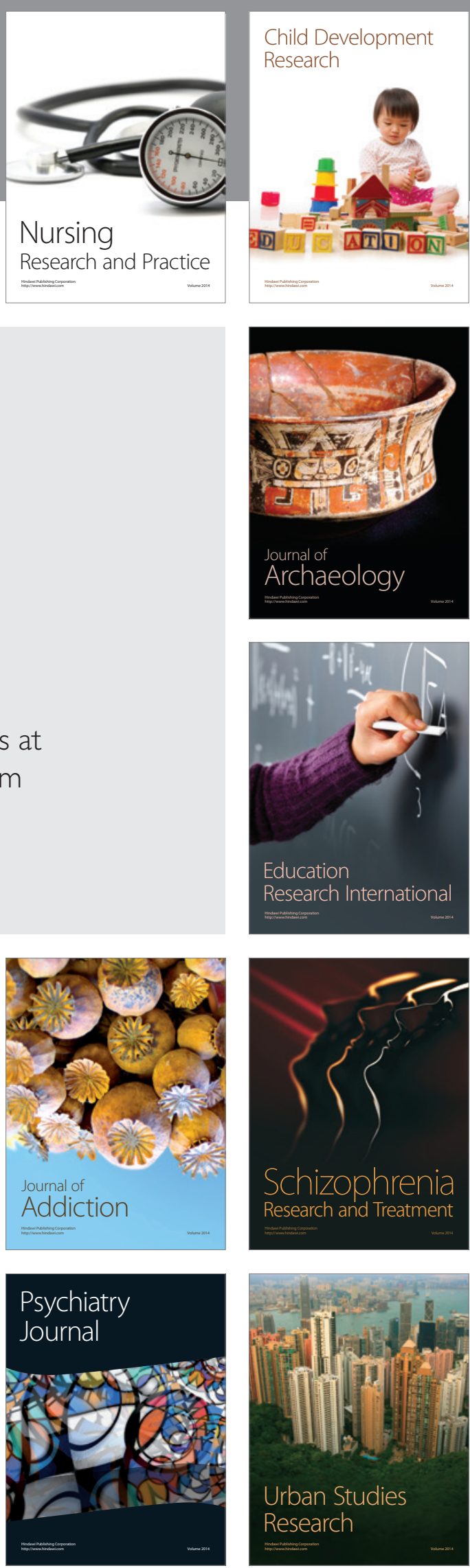